\title{
Synthesis of high yield and highly graphitic multi walls carbon nanotubes from plastic waste without the use of external catalyst
}

\author{
Pranav K. Tripathi*, Shane Durbach and Neil J. Coville \\ DST-NRF Centre of Excellence in Strong Materials and Molecular Sciences Institute, School of \\ Chemistry, \\ University of the Witwatersrand, WITS, Johannesburg 2050, South Africa \\ *Email: ptripathi2@gmail.com, pranav.tripathi@gmail.com
}

Plastic garbage is a widely considered source of pollution. As estimated in 2010, 192 countries have generated 275 million metric tons (MT) of plastic waste [1] and these plastic wastes generally composed of HDPE, LDPE, and PP etc. For years, researchers have been looking for various ways to overcome this problem of the long term disposal of such a large quantity of plastic waste, as current processes are not environmentally favorable. On the other hand, carbon nanomaterial's (CNMs) such as nanotubes (CNTs), fibers (CNFs) and spheres (CNSs) are materials with extraordinary physical and chemical properties, which often have energy and resource intensive production processes. In recent years, some researchers have suggested the idea of using plastic polymers as a carbonaceous feed for CNTs and CNFs production. The studies to date are rather scattered [2]. In this work, we have successfully synthesized high yield and highly graphitic multi walls carbon nanotubes (MWCNTs) and CNSs from our own laboratory plastic waste. MWCNTs and CNSs were synthesized in two stages in a stainless steel (SS) 316 metal tube and quartz tubing based CVD furnace respectively and under nitrogen flow and at temperature from 700 to $1200^{\circ} \mathrm{C}$ at $100^{\circ} \mathrm{C}$ differences. Interestingly, MWCNTs were synthesized successfully from plastic waste without the use of an external catalyst source in SS 316 metal tubing and MWCNTs had a very high graphitic nature, as depicted by their Raman spectra (Figure 1). While in quartz tube we failed to get MWCNTs without any catalyst from plastic waste and obtained CNSs. We believe that MWCNTs growth process from plastic waste in a SS 316 metal tube is due to a 'dusting' process and in quartz tube CNSs were formed due to decomposition of the carbon source at very high temperatures. The yields of MWCNTs were very high (ca. $56 \%$ ) at $900^{\circ} \mathrm{C}$ while the CNSs yield at $1100^{\circ} \mathrm{C}$ (ca. $14 \%$ ). The

method of MWCNTs synthesis using plastic waste can reduce the environmental impact of plastic waste and will offer highly valuable product via cost effective manner.

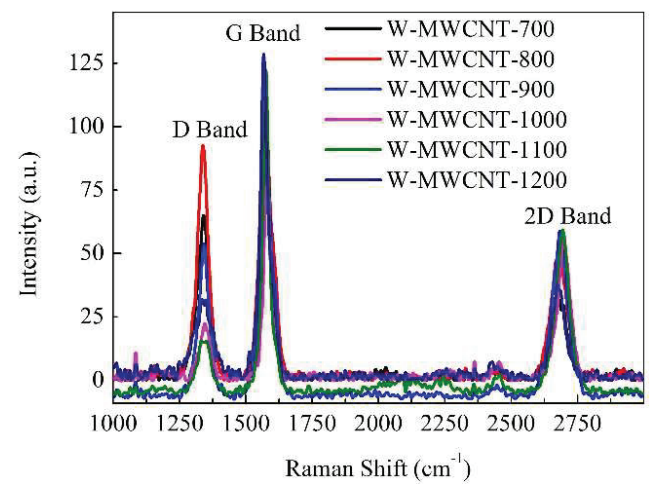

Figure 1: Raman spectra of the MWCNTs synthesized without the use of external catalyst and plastic waste as carbon source

\section{References}

1. Jenna, J., Roland, G., Chris, W., Theodore, S., Miriam P., Anthony A., Ramani N., and Kara L. (2015), Plastic waste inputs from land into the ocean, Science, 347, 768-771.

2. Bazargan, A., McKay G. (2012), A review Synthesis of carbon nanotubes from plastic wastes. Chem. Eng. J. 195-196, 377-391. 